\title{
Bone marrow-derived recipient cells in murine transplanted hearts: potential roles and the effect of immunosuppression
}

\author{
Nana Rezai ${ }^{1}$, Stephane Y Corbel ${ }^{2}$, Darya Dabiri ${ }^{1}$, Alexandra Kerjner ${ }^{1}$, Fabio MV Rossi ${ }^{2}$, \\ Bruce M McManus ${ }^{1}$ and Thomas J Podor ${ }^{1}$ \\ ${ }^{1}$ Department of Pathology and Laboratory Medicine, The James Hogg iCAPTURE Centre for Cardiovascular \\ and Pulmonary Research, St Paul's Hospital-University of British Columbia, Vancouver, BC, Canada and \\ ${ }^{2}$ Biomedical Research Centre, University of British Columbia, Vancouver, BC, Canada
}

\begin{abstract}
Currently, there is intense debate regarding the origin of reparative cells in injured hearts and vasculature. To determine the contribution of recipient bone marrow (BM)-derived cells to the regeneration of cells in the vasculature of transplanted hearts and to examine the effect of immunosuppression on this phenomenon, we evaluated the fate of green fluorescent protein (GFP)-positive recipient BM cells in non-GFP-expressing cardiac allografts. C57BL/6 BM-GFP chimeric recipients underwent cardiac transplantation. Allografts were immunosuppressed with tacrolimus for $\mathbf{1 4}$ or $\mathbf{3 0}$ days post-transplantation or were saline treated. Hearts were excised and stained with markers for endothelial cells (EC) or smooth muscle cells (SMC). Colocalization with BMderived recipient cells was evaluated using confocal microscopy with three-dimensional image analysis. Immunosuppression with tacrolimus did not affect the frequency of recipient BM-derived cell chimerism as EC or SMC phenotypes. A higher frequency of EC chimerism was found at 14 days as compared to 30 days posttransplantation in allograft hearts. BM-derived recipient cells are recruited to areas of donor vascular injury with intercalation of recipient EC and SMC in the setting of ongoing alloimmune recognition of the allograft. Our findings confirm that immunosuppression with tacrolimus does not affect the frequency of recipient BMderived cell repopulation at an early time point 14 days post-transplantation. EC repopulation by BM-derived recipient cells was found to be an early event in transplanted allograft hearts, which decreased in frequency over time.

Laboratory Investigation (2005) 85, 982-991. doi:10.1038/labinvest.3700302; published online 20 June 2005
\end{abstract}

Keywords: bone marrow; transplant heart; immunosuppression; endothelial cell; smooth muscle cell

Heart transplantation is a life-prolonging procedure that has become an accepted surgical treatment for patients with end-stage congestive heart failure. Despite improvements in outcomes over the last two decades, both acute and chronic rejection continue to limit survival and quality of life in heart transplant recipients. ${ }^{1}$ Transplant vascular disease (TVD), as an expression of chronic rejection, is a rapidly progressive form of atherosclerosis that occurs in the vessels of all solid organ transplants, including the heart. Cardiac TVD is estimated to

Correspondence: Dr TJ Podor, PhD, Department of Pathology and Laboratory Medicine, The James Hogg iCAPTURE Center for Cardiovascular and Pulmonary Research, St Paul's HospitalUniversity of British Columbia, Rm 161, 1081 Burrard St., Vancouver, BC, Canada V6Z 1Z3.

E-mail: tpodor@mrl.ubc.ca

Received 4 March 2005; revised and accepted 5 May 2005; published online 20 June 2005 affect more than $40 \%$ of recipients who survive beyond four years after transplantation. ${ }^{1,2}$

Recent studies have suggested that undifferentiated progenitor cells may emigrate from the recipient to the graft, contributing to vascular remodeling and possibly contributing to the atherosclerotic process of TVD. ${ }^{3-5}$ However, the degree of recipient cell repopulation of cells of the vessel wall is currently a matter of intense debate ${ }^{6}$ since there are discrepancies in reported rates of chimerism in damaged vessels and hearts. ${ }^{3-5,7-10}$ These discrepancies may be attributed to confounding factors, including different assays and techniques used to detect cell differentiation or colocalization events, as well as variations in models used to examine chimerism rates.

To address the fate of endogenous recipient bone marrow (BM)-derived progenitor cells in the vasculature of transplanted (Tx) hearts, we performed heterotopic heart transplantation using chimeric 
recipient mice expressing green fluorescent protein (GFP)-positive BM in order to track the migration of the BM-derived recipient cells. Moreover, we measured the effect of immunosuppression vs none on the frequency of recipient GFP-labeled BM-derived endothelial cells (EC) and smooth muscle cells (SMC) in the coronary arteries of the Tx heart.

\section{Materials and methods}

\section{Generation of BM-Chimeric Mice}

The study protocol was approved by the University of British Columbia Office of Research Services Committee on Animal Care. C57BL/6 (CD45.2) and C57BL/6 (GFP/CD45.1) mice were bred in-house and maintained in a pathogen-free environment. The C57BL/6 (GFP/CD45.1) mice express GFP driven by the CMV enhancer-chicken beta-actin hybrid promoter. They were a kind gift of Dr I Weissman. ${ }^{11}$ C57BL/6 (GFP/CD45.1) mice (8 weeks old) were used as donors and 8- to 10-week-old C57BL/6 (CD45.2) mice as recipients.

\section{Cells}

$\mathrm{BM}$ was flushed out of the femurs and the tibias of C57BL/6 (GFP/CD45.1) mice and single-cell suspensions were made. Red cells were lysed, cells were enumerated and $5 \times 10^{6}$ cells were injected into the tail vein of lethally (950 rad) irradiated recipient C57BL/6 mice.

\section{Peripheral Blood Analysis of BM-Chimeric Mice}

At various time points after transplantation, $150 \mu$ l of peripheral blood were collected from the tails of recipient BM-chimeric mice. Reconstitution of the hematopoietic system was analyzed by flow cytometry on a FACScan (Becton Dickinson) using a protocol as described previously. ${ }^{7}$ Mice with more than $80 \%$ of GFP-positive peripheral blood leukocytes were used as recipient mice for the heterotopic heart transplant experiments 2 months after BM transplantation.

\section{Heterotopic Cardiac Transplantation}

Heterotopic cardiac transplantation was performed as described. ${ }^{12}$ Hearts of 10 -week-old $129 \mathrm{SV} / \mathrm{j}$ mice were transplanted into the reconstituted GFP-BMchimeric C57BL/6 mice described above. Control syngrafts consisted of 10-week-old C57BL/6 donor hearts transplanted into C57BL/6 GFP-BM-chimeric mice. Allograft mice were treated with tacrolimus (Fujisawa, Osaka, Japan) administered intraperitoneally (i.p.) $(6 \mathrm{mg} / \mathrm{kg})$ daily for a period of $14(n=5)$ and $30(n=5)$ days post-transplantation. A subset of allograft mice did not receive any immunosuppressive therapy, and were treated with daily i.p. injections of saline $(n=5)$ for a period of 14 days post-transplantation. Mice were euthanized at 14 and 30 days post-transplantation.

\section{Immunofluorescence and Histochemical Staining}

Native (Nat) and Tx hearts were excised from the allograft and control syngraft groups and routinely processed for cryosections. Immunofluorescence staining was performed using a modified protocol as described previously ${ }^{13}$ and other sections were stained with hematoxylin and eosin (H\&E). Briefly, hearts in all treatment groups were perfusion fixed with $2 \%$ paraformaldehyde and OCT embedded. Frozen midventricular sections were incubated with primary antibodies, anti-smooth muscle (SM) $\alpha$-actin (DAKO, Carpinteria, CA, USA), von Willebrand factor (vWf) (DAKO) and anti-CD45 (BD Pharmingen, Mississauga, ON, Canada). Alexa 594-conjugated secondary antibody was used and nuclei were counterstained with Hoechst 33342 (Molecular Probes, Eugene, OR, USA).

\section{Confocal Microscopic Analysis and Quantitation of the Recipient BM-Derived Cells}

All images were obtained using a Leica AOBS $^{\mathrm{TM}}$ SP2 confocal microscope. In all, $10 \mu \mathrm{m}$ sections of the Tx and Nat hearts were stained for vWf, SM $\alpha$-actin and CD45, and analyzed. The number of GFP-positive BM-derived recipient cells in the hearts was quantitated at 14 and 30 days post-transplantation in all allograft and syngraft groups. Briefly, 10 representative microscopic fields at $1.4 \times 10^{-3} \mathrm{~mm}^{3}$ from the same anatomical location in each myocardial section were taken and Image-Pro ${ }^{\circledR}$ Plus software (MediaCybernetics ${ }^{\mathbb{R}}$, San Diego, CA, USA) was used to count the number of nuclei and nucleated GFPpositive recipient cells in the confocal optical sections. For vWf and SM $\alpha$-actin quantitation, analysis for colocalization with BM-derived recipient GFP-positive cells was performed on volumes of $5.3 \times 10^{-4} \mathrm{~mm}^{3}$ optical stacks of vessels, $60-200 \mu \mathrm{m}$ wide in diameter, found in representative midventricular sections from Tx and Nat hearts. Stacks were reconstructed using Volocity ${ }^{\mathrm{M}}$ (Improvision ${ }^{\mathbb{R}}$, Boston, MA, USA) and Metamorph ${ }^{\circledR}$ (Universal Imaging Corporation $^{\mathrm{TM}}$, Downingtown, PA, USA) image softwares. Two independent, blinded observers reviewed each reconstructed vessel. Nucleated recipient BMderived GFP-positive cells seeded within the vessel walls were quantitated and the number of morphologically distinguishable cells that stained dual positive for GFP and either vWf or SM $\alpha$-actin in the vessel wall was scored. Cells were considered endothelial in origin if they were positioned on the luminal side of the vessel, thin and elongate in shape, and demonstrated intracellular granular vWf staining. Cells were considered vascular SMC if they were in the subendothelium or media, were spindle shaped, blunt ended and exhibited intracellular SM $\alpha$-actin 
staining. Each chimerism count was expressed as a percentage of total EC or SMC, respectively. In addition, to confirm the presence of GFP within individual cells separated from the autofluorescent background, spectrophotometric wavelength lambda scanning was performed on tissue sections. Algorithms were then applied to further separate the spectral fingerprint for GFP (510 nm emission maximum) from the autofluorescence fingerprint (535$545 \mathrm{~nm}$ emission maximum) in order to ensure accurate recipient BM-derived GFP cell detection.

\section{Statistical Analyses}

Data for the quantitation of recipient cell seeding are given as mean \pm s.e.m. Comparison between groups was made using ANOVA and a post hoc Tukey's test was used to determine statistical differences between groups. The frequency of vWf or SM $\alpha$-actin and GFP double-positive cells in the vessel wall are shown as mean \pm s.e.m. of the total recipient EC or SMC. A $P$-value of less than 0.05 was considered significant.

\section{Results}

Recipient Cell Seeding to the Tx Heart and the Effect of Immunosuppression

We utilized chimeric GFP-BM recipient mice in a heterotopic heart transplant model to identify the extent of contribution of recipient BM-derived cells to the repopulation of damaged cells in the vessels of Tx hearts. Our data demonstrate the presence of infiltrating BM-derived recipient cells in the Tx hearts of both immunosuppressed and nonimmunosuppressed allograft mice at both 14 and 30 days post-transplantation (Figure 1). BMderived recipient cell seeding to the Tx heart was significantly greater $(P<0.05)$ as compared with the Nat heart controls in allograft groups receiving immunosuppression at both $14(\mathrm{Tx}=24 \pm 2.0 \%$, Nat $=5.2 \pm 1.0 \%)$ and $30(\mathrm{Tx}=23 \pm 3.6 \%$, Nat $=$ $4.3 \pm 1.1 \%$ ) days post-transplantation (Figure 2). Syngraft controls showed minimal recipient BMderived cell seeding to the Tx and Nat hearts at both 14 days $(\mathrm{Tx}=2.7 \pm 0.59 \%$, Nat $=3.5 \pm 2.2 \%)$ and 30 days $(\mathrm{Tx}=6.6 \pm 0.46 \%$, Nat $=6.8 \pm 4.0 \%)$ post-transplantation. At 14 days post-transplantation, the total BM-derived recipient cell seeding was found to be significantly higher $(P<0.05)$ in the Tx hearts of non-immunosuppressed allografts $(41 \pm 2.1 \%)$ as compared with immunosuppressed allografts $(24 \pm 2.0 \%)$.

\section{CD45 Expression of Recipient BM-Derived Cells}

Allograft and syngraft hearts were stained for the panleukocyte marker CD45 to identify the inflammatory cell proportion of the recipient GFP-positive BM-derived cells, which seeded to the cardiac interstitium and vasculature. The results (Figure 3)

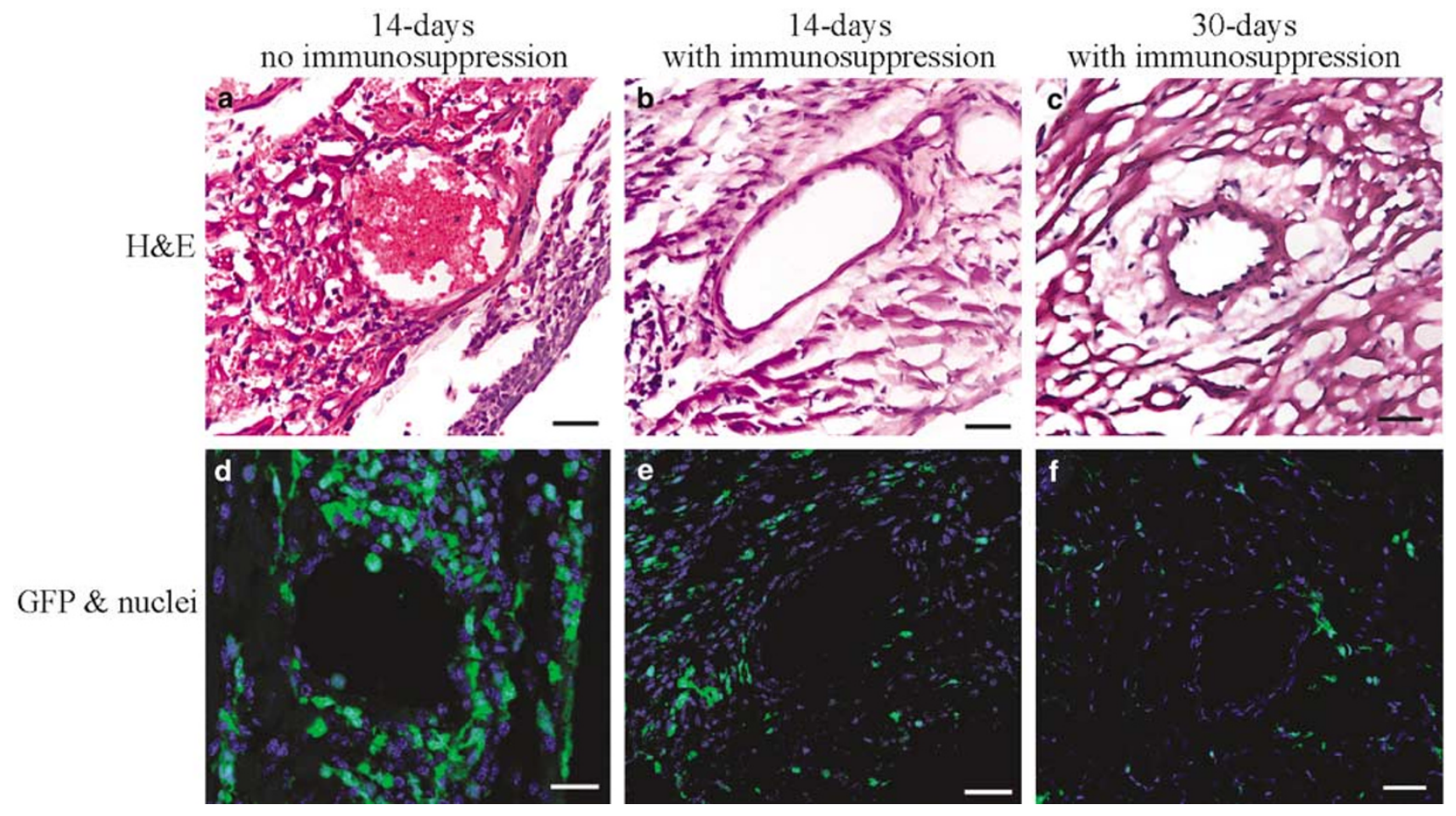

Figure 1 Recipient BM-derived cell seeding to Tx hearts of allografts. H\&E staining of allograft Tx hearts at 14 days post-transplantation without tacrolimus (a), with tacrolimus (b) and 30 days post-transplantation with tacrolimus (c). Serial sections of Tx hearts were stained with Hoechst 33342 to identify nuclei (blue) and the recipient GFP-positive BM-derived cells (green) (d-f). Scale bars: $30 \mu \mathrm{m}$. 


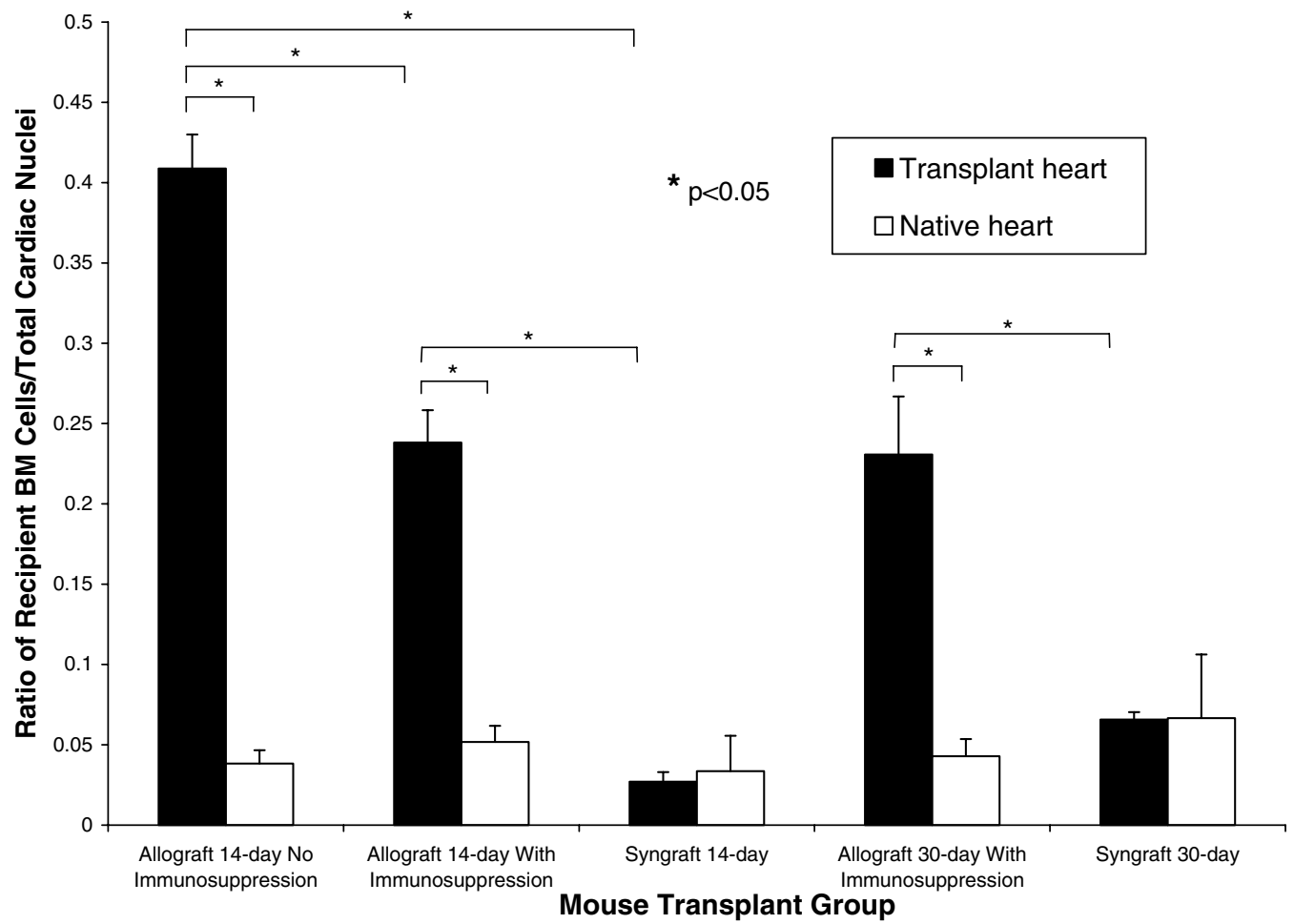

Figure 2 Quantification of recipient BM-derived GFP-positive cell seeding. GFP-positive recipient BM-derived cells seeded within Tx and Nat hearts of all allograft and syngraft groups were quantified and expressed as a fraction of total cardiac nuclei and graphically represented as the mean \pm s.e.m. from animals per group. ${ }^{*}$ Significant difference, $P<0.05$.
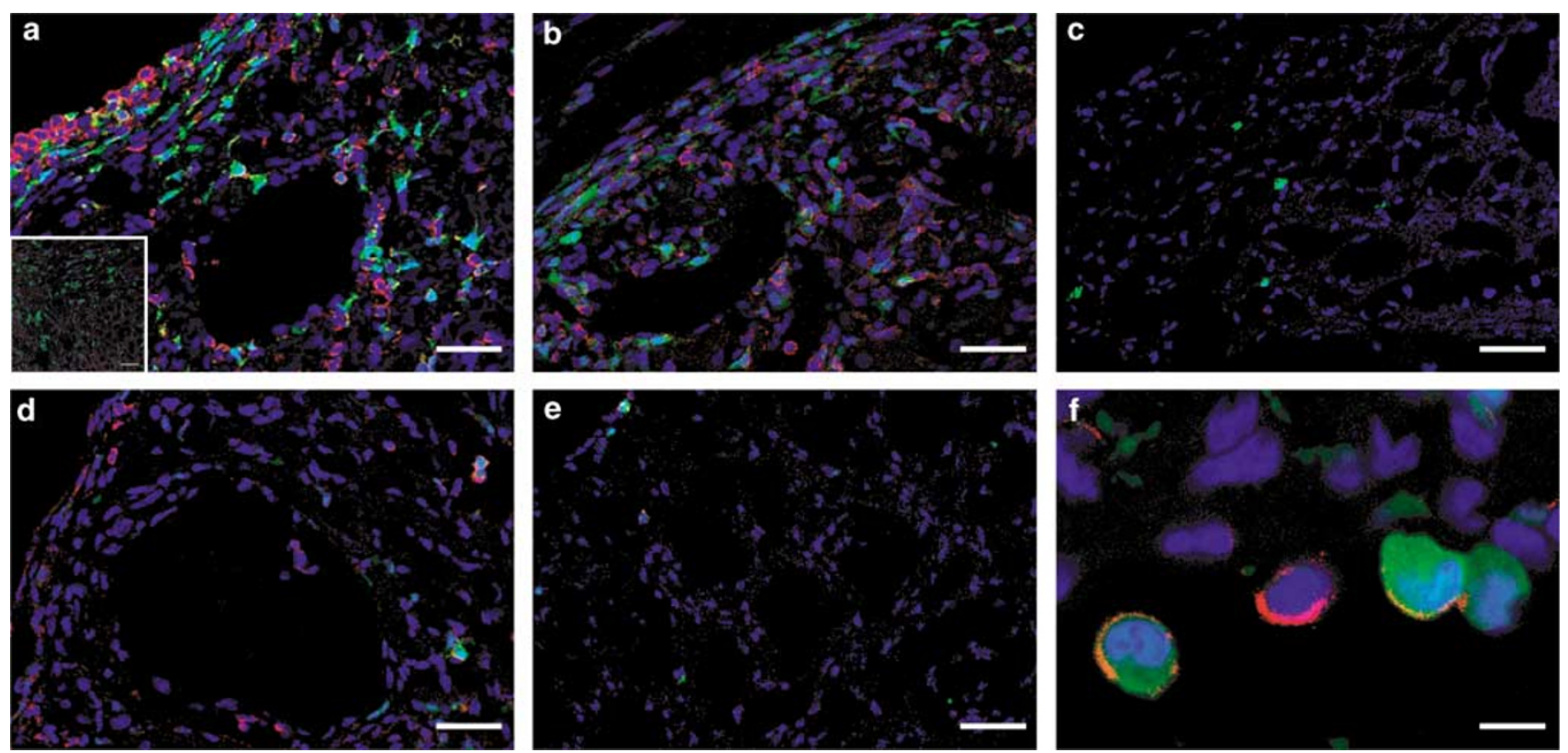

Figure 3 vWf staining of EC in Tx hearts. Confocal micrographs of (a, f and h) allograft vessels. (b) Hoechst 33342 nuclear staining (blue), (c) GFP-positive recipient BM-derived cells (green), (d) vWf-positive EC (red) and (e, g and i) merged images at higher magnification show dual-positive granular staining for vWf within the GFP cytoplasm of some cells (arrows). Inset (a) shows control immunostain with specific antibody isotype control. Scale bars $=$ (a) $40 \mu \mathrm{m}$, (b-e and g) $10 \mu \mathrm{m}$ and (f) $20 \mu \mathrm{m}$.

indicate that in the Tx hearts, CD45 was coexpressed by a large proportion of the GFP-positive BMderived recipient cells found in the perivascular space and cardiac interstitium in allograft hearts at 14 and 30 days post-transplantation, both with and without immunosuppression. 


\section{EC Differentiation in Tx Hearts}

In all Tx hearts of allograft groups, we found GFPvWf dual-positive cells in the endothelial lining of vessels (Figure 4). The frequency of GFP-vWf dualpositive cells out of the total EC within the vessel wall was $11.8 \pm 2.5$ and $4.0 \pm 1.2 \%$ for 14 and 30 days post-transplantation, respectively (Table 1). The results indicate that the frequency of differentiation events for recipient BM-derived cells into an EC phenotype within the vessel wall of Tx hearts appears to be higher at 14 days post-transplantation than at 30 days $(P=0.04)$. The frequency of GFPvWf dual-positive cells was similar in the vessels of Tx hearts in both the non-immunosuppressed $(9.4 \pm 2.1 \%)$ and the immunosuppressed (11.8 \pm $2.5 \%)$ allograft groups at 14 days post-transplantation. These findings suggest that 14 days of immunosuppressive therapy does not significantly affect the frequency of recipient BM-derived cell chimerism in vessels of Tx hearts. Nat hearts demonstrated a low level of GFP-vWf dual-positive cells only in the vessels of non-immunosuppressed allograft hearts, and no dual-positive cells were found in any other allograft or syngraft recipient Nat hearts.

\section{SMC Differentiation in Tx Hearts}

SM $\alpha$-actin was used as a phenotypic marker for vascular SMC in Tx and Nat hearts. The results (Table 1) indicate that at 14 days post-transplantation, the frequency of SM $\alpha$-actin-GFP dual-positive cells out of the total number of SMC within the vessel wall is not significantly different in the Tx hearts of the allograft groups (nonimmunosuppressed $=0.9 \pm 0.89 \%$ and immunosuppressed $=2.0 \pm 2.0 \%$ ). This suggests that the frequency of differentiation events into an SMC phenotype is not affected by immunosuppressive treatment with tacrolimus (Figure 5). Further, there appears to be no significant difference in the number of SM $\alpha$-actin-GFP dual-positive cells in Tx hearts of immunosuppressed allografts at 14 and 30 days post-transplantation $(2.0 \pm 2.0$ and $0.4 \pm 0.4 \%$, respectively). No vessels in the Nat heart controls contained SM $\alpha$-actin-GFP dual-positive cells.

\section{Discussion}

TVD is one of the leading causes of graft failure and death after the first perioperative year and is now the most important problem in clinical organ transplantation. The original concept of TVD and vascular remodeling assumed that vascular EC and SMC in TVD originate from graft tissue and are therefore donor derived. ${ }^{1,14}$ In recent years, several groups have reported data both supporting, and discounting ${ }^{17,18}$ repopulation of graft vessels by recipient-derived cells after solid organ transplantation. However, several questions remain unanswered, including: (1) whether there is significant repopulation of cells of the vessel wall with recipient-derived cells, and if so, what the origin of these cells is, (2) if the repopulation of vascular cells is either an early or late event, or a continuous process throughout TVD progression and (3) what the effect of immunosuppression is on recipient cell repopulation of cells of the vessel wall of Tx hearts. The possibilities for the origin of recipient cells recruited to damaged vessels include circulating vessel wall-derived adult EC, tissue resident progenitor cells or recipient BM-derived cells. In this study, we investigate these questions by elucidating the contribution of recipient BM-derived cells in the repopulation of cells of the vessel wall at both an early (14 days) and late (30 days) time-point posttransplantation as well as examining the effect of immunosuppressive therapy with tacrolimus on the frequency of recipient BM-derived cell differentiation into vascular cell phenotypes.

The results of this study support the concept that recipient BM-derived cells migrate and localize to the Tx heart as early as 14 days post-transplantation. Although these recipient BM-derived cells predominantly consisted of inflammatory cell subsets, we were particularly interested in examining the role of non-inflammatory recipient cell populations found to seed sites within vessels of the Tx hearts. Our results, using quantitative image analysis, indicate that recipient BM-derived cells are recruited to vessels of the allograft Tx heart where they participate in the replacement of EC, as well as SMC at a very low frequency. EC replacement in Tx hearts by recipient BM-derived cells at 14 days post-transplantation was significantly greater as compared with 30 days post-transplantation. It is well established that immune-mediated damage of donor EC is believed to be an initiating event in TVD. TUNEL labeling in coronary arteries affected by TVD has revealed apoptotic cells in these lesions and electron microscopic documentation of denuded donor EC in animal models of this disease indicate that large-scale unregulated damage is abundant in TVD. ${ }^{19,20}$ The resultant damage likely contributes to TVD by increasing vascular permeability, fostering the infiltration of plasma proteins into damaged blood vessels and the myocardial interstitium. Consequently, this donor EC damage and cell

Figure 4 Hematopoietic phenotype of GFP-positive recipient BM-derived cells in Tx hearts. Confocal micrographs of (a) nonimmunosuppressed and (b) tacrolimus-treated allografts 14 days post-transplantation and (d) tacrolimus-treated allografts 30 days posttransplantation reveal a population of recipient BM-derived cells (green), which stain positively for CD45 (red). (c and e) Controls 14 and 30 days post-transplantation, respectively. (f) Higher magnification of GFP-positive recipient BM-derived cells. Nuclei (blue) stained with Hoechst 33342. Inset (a) shows control immunostain with specific antibody isotype control. Scale bars: (a-e) $50 \mu \mathrm{m}$ and (f-i) $10 \mu \mathrm{m}$. 
Non-immunosuppressed 14 days post-transplantation

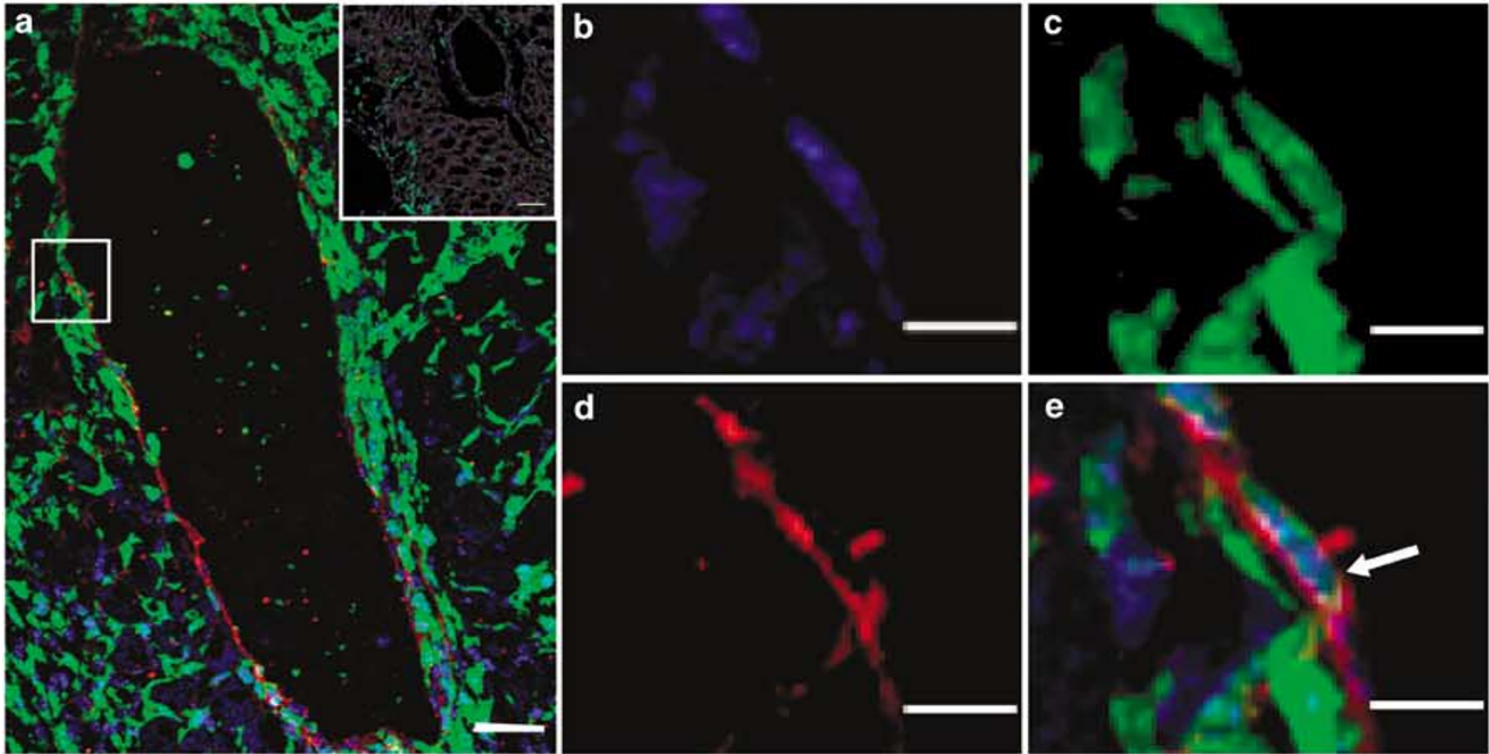

Immunosuppressed 14 days post-transplantation

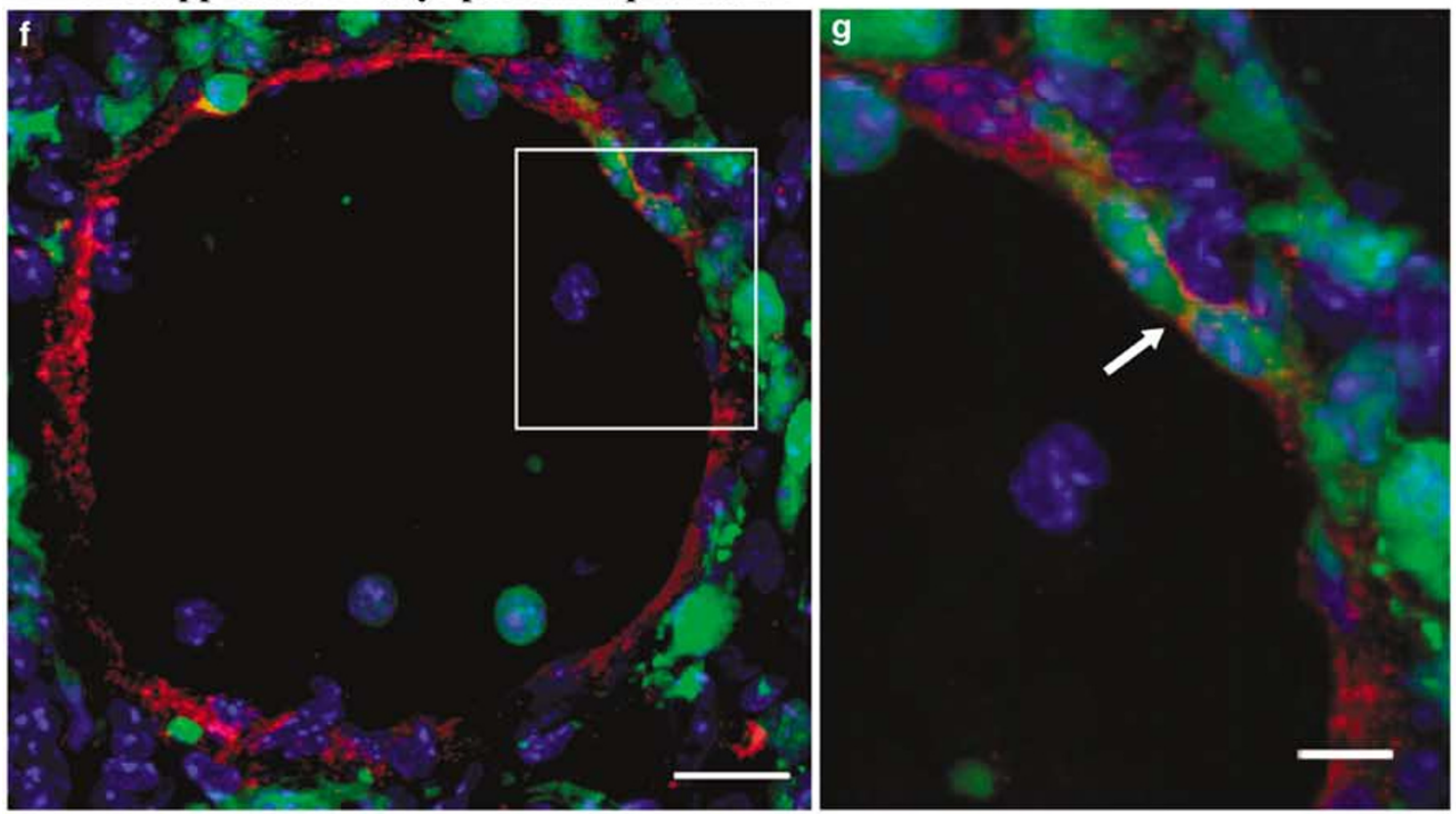

Immunosuppressed 30 days post-transplantation

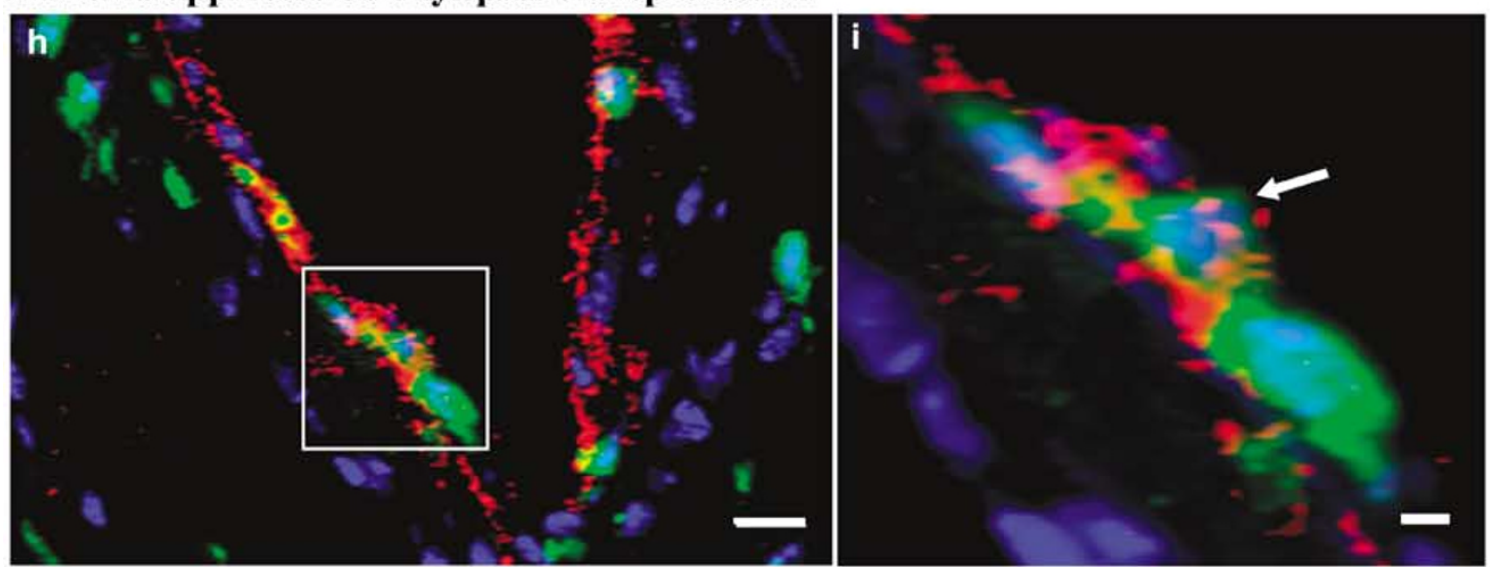


death early post-transplantation may lead to the release of a variety of cytokines and other signals in the vessel wall, which in turn lead to the homing of recipient BM-derived cells. The precise signals that attract these recipient BM-derived cells to the sites of vascular damage in TVD remain unclear. This early EC apoptosis in TVD may account for why there is a decrease in EC replacement in the vessels of $\mathrm{Tx}$ hearts from 14 to 30 days posttransplantation.

SMC chimerism was consistently at a low level over time (14 and 30 days post-transplantation). As well, at the 14-day time point, the results suggest that immunosuppression with tacrolimus does not significantly affect the EC and SMC replacement by recipient $\mathrm{BM}$-derived cells in the $\mathrm{Tx}$ heart of allografts. Moreover, the higher levels of BM-derived recipient cell chimerism found in Tx as compared to
Nat control hearts supports the concept of preferential seeding of these cells to sites of vascular damage.

Uncovering the true extent of EC and SMC replacement by recipient cells in TVD is an important biological objective that has yet to be addressed adequately. In order to determine the precise contribution of recipient cells to EC and SMC replacement in TVD, it is vital to utilize and compare findings between the same models of vascular disease, that is, cardiac allograft vasculopathy, as other models in which there is endothelial injury may lead to variable findings due to differences in pathogenetic mechanisms. Our study depends on a well-established heterotopic cardiac allograft model to study the role of recipient BMderived cells in EC and SMC replacement. However, even in similar animal models of TVD, there

Table 1 Chimerism frequencies of recipient cells in vessel walls of Tx and Nat hearts

\begin{tabular}{|c|c|c|c|c|}
\hline \multirow[t]{2}{*}{ Transplant group } & \multicolumn{2}{|c|}{$E C$} & \multicolumn{2}{|c|}{$S M C$} \\
\hline & $T x$ & Nat & $T x$ & Nat \\
\hline Allograft 14 days with tacrolimus & $11.8 \pm 2.5 \%$ * & 0 & $2.0 \pm 2.0 \%$ & 0 \\
\hline Allograft 14 days no tacrolimus & $9.4 \pm 2.1 \%$ & $2.0 \pm 2.0 \%$ & $0.90 \pm 0.89 \%$ & 0 \\
\hline Syngraft 14 days & 0 & 0 & 0 & 0 \\
\hline Allograft 30 days with tacrolimus & $4.0 \pm 1.2 \%$ * & 0 & $0.4 \pm 0.4 \%$ & 0 \\
\hline Syngraft 30 days & 0 & 0 & 0 & 0 \\
\hline
\end{tabular}

Mean \pm s.e.m.; $\mathrm{Tx}=$ transplanted heart; Nat $=$ native heart.

${ }^{*} P=0.04$.
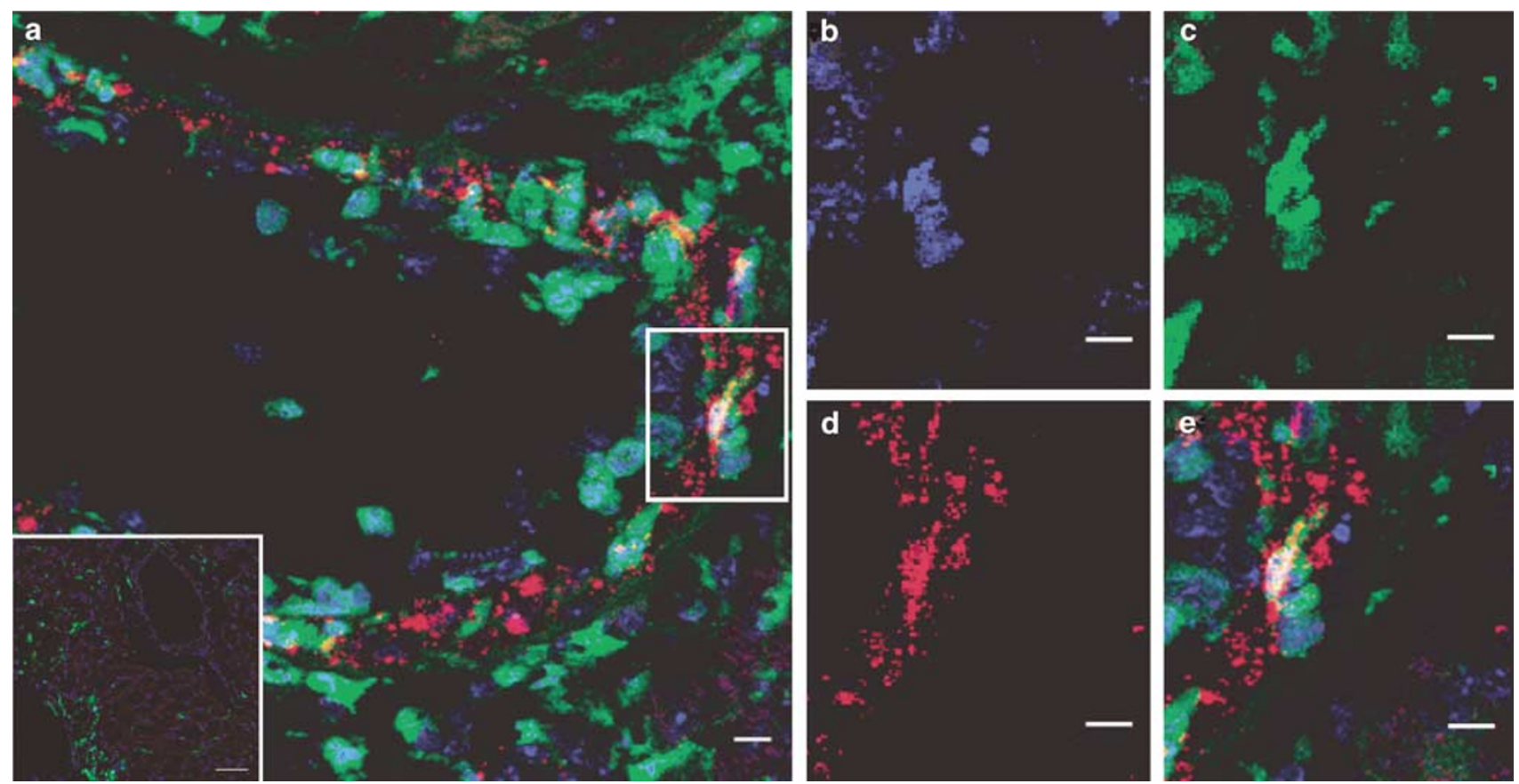

Figure 5 SM $\alpha$-actin staining in vessels of allografts. Confocal micrographs of (a) Tx heart 14 days post-transplantation. (b) Hoechst 33342 nuclear staining (blue), (c) GFP-positive recipient BM-derived cells (green), (d) SM $\alpha$-actin-stained SMC (red) and (e) merged at higher magnification reveal green/red dual-positive staining of rare recipient cells. Inset (a) shows control immunostain with specific antibody isotype control. Scale bars $=(\mathbf{a}-\mathbf{e}) 10 \mu \mathrm{m}$. 
remains large discrepancies and debate regarding the frequency of recipient cell replacement of cells of the vessel wall and particularly the contribution of BM-derived cells. Some studies report that almost all cells in the vessel wall are recipient derived in models of aortic transplantation, ${ }^{3,16,21,22}$ vein grafting $^{23}$ and cardiac allografts, ${ }^{4,22}$ while other studies have reported contrary results suggesting that recipient cells only contribute minimally, if at all, to vascular cell replacement in similar models of allograft vasculopathy. Attempts at explaining these discrepancies have suggested that immunosuppressants may potentially affect the pathogenesis of TVD, while others suggest that certain models may underestimate the potential contribution of recipient cells in vascular remodeling. Furthermore, the contribution may vary in different tissues as suggested by one study showing that recipient-derived EC replaced donor endothelium in aortic but not cardiac allografts. ${ }^{22}$

Recent studies propose that imaging artifacts related to the lack of stringent methodological approaches may explain the major discrepancies in the reported contribution of BM-derived recipient cells as a major source of SMC in graft vasculopathy $\left(5 \%{ }^{21}-82.5 \%{ }^{4}\right) .{ }^{24}$ We have utilized more rigorous imaging techniques and analytical strategies to confirm the specificity of the GFP spectral tracking and three-dimensional imaging than those employed before when investigators have reported both high $^{4}$ and low ${ }^{21}$ rates of EC and SMC replacement by BM-derived recipient cells. Several studies have utilized both conventional light microscopy ${ }^{3,10,25}$ and confocal microscopy $y^{4,9}$ to examine the fate of recipient cells within Tx hearts. With respect to confocal microscopy, although this technique does allow the power to analyze thicker tissue sections as compared to conventional light microscopy, there is also a risk in relying solely on the color overlap of two fluorophores in a given thickness of tissue to determine the phenotype of cells of interest. Confocal microscopy increases the capability of distinguishing adjacent structures in thicker sections of tissue if it is utilized to its full potential. By obtaining optical sections of a given tissue, confocal microscopy enables users to produce three-dimensional reconstructions and volume renderings in order to examine target cells in that given volume of tissue in a three-dimensional, more accurate view. In this study, we have used $10 \mu \mathrm{m}$ sections and obtained $0.4-0.5 \mu \mathrm{m}$ optical sections of tissue stained for EC and SMC markers. These optical sections were then reconstructed to produce a volume rendering of each vessel and were examined by segmentation analysis of the three color channels and by rotating the volume through $180^{\circ}$ at increments of $1^{\circ}$. In this manner, we anticipate accurate determination of colocalization events between cellular markers in the exact three-dimensional plane ( $x, y$ and $z$ planes) of the tissue. In addition, a wavelength (lambda) scan of the GFP-expressing recipient BM-derived cells was performed using a confocal laser scanning microscope to confirm the presence or absence of GFP within individual cells. We believe the present observations bring us closer to the true frequency of recipient BM-derived cell chimerism in TVD.

Thus, although our results for the frequency of differentiation events into both an EC and SMC phenotype in murine heart allografts is consistent with some recent findings, ${ }^{25,26}$ we report lower rates of recipient cell replacement of EC and SMC in cardiac allografts as compared to other studies, which utilized confocal microscopic analysis. ${ }^{3,4,9}$ As mentioned above, these lower rates may be attributed to our utilization of a rigorous method of image analysis for all tissue sections, thereby enhancing our identification of true EC and SMC of recipient $\mathrm{BM}$ origin, and diminishing the chance of both falsely identifying inflammatory cells as EC or SMC in the vessel wall and falsely identifying subadjacent cells as colocalization events. Another factor that may contribute to our lower rates of recipient cell chimerism is our selective examination of only BM-derived recipient cell contributions to chimerism in the vessels of Tx hearts. Indeed, the $\mathrm{BM}$ is likely not the only source of cells that contribute to this phenomenon and numerous reports have in fact shown that vascular cells are heterogeneous and that both EC and SMC in vascular lesions are composed of cells of diverse origins. ${ }^{27,28}$ In addition, there may be a population of radiation-resistant $\mathrm{BM}$ progenitor cells that also contribute to vascular remodeling, which would not be labeled with the GFP marker following BM reconstitution, and thus, would not be detected by our stringent image analysis technique.

To address the question of whether immunosuppression may affect the recipient cell repopulation of EC and SMC in TVD, we compared the effect of immunosuppressive treatment with tacrolimus vs no treatment on the rates of recipient BM-derived cell chimerism in the vessels of our heterotopic heart transplant model. Our findings confirm that even as early as 14 days post-transplantation, immunosuppression in a cardiac allograft model does not significantly affect the frequency of recipient BM-derived cell repopulation of EC and SMC. Hence, our data suggest that discrepancies in the reported rates of recipient cell chimerism at early time points in vessels of Tx hearts to date are not due to an effect of immunosuppressive treatment on progenitor cell populations in the BM.

To our knowledge, this study is among the first to examine the effect of immunosuppressive therapy on BM-derived recipient cell chimerism in the Tx heart. Interestingly, we found that although immunosuppression with tacrolimus led to a significant decrease in the total number of BM-derived recipient cells seeding to sites within the Tx heart of allografts at 14 days post-transplantation, it did not appear to affect the frequency of recipient 
BM-derived cell repopulation of EC and SMC. One possible reason for this is that the time point used in this study was not long enough to see differences in chimerism rates between the immunosuppressed vs the non-immunosuppressed allograft hearts. The murine heterotopic heart transplant model utilized in this study does not allow survival of the Tx heart past 14 days without immunosuppressive therapy, as the non-immunosuppressed Tx heart ceases to beat past this time period, and it is possible that we would begin to see differences in rates of chimerism due to immunosuppression at the 30 day time point. However, there is a possibility that a progenitor cell population within the BM is not affected by immunosuppressive therapy with tacrolimus, and that this treatment only influences the inflammatory cell response to the heart of allografts.

The findings reported here are novel in the field of cardiac TVD and the study of vascular repopulation events. This is the first report which demonstrates that immunosuppression with tacrolimus does not affect the frequency of repopulation of damaged vessels in the donor heart by recipient BM-derived cells in a murine model of TVD. We demonstrate that EC replacement is an early event, found to occur at 14 days post-transplantation, but at a rate that diminishes with time up to 30 days post-transplantation. We found that the frequency of recipient BMderived cell chimerism events at an early time point of 14 days post-transplantation, into both EC and SMC phenotypes, remains constant irrespective of immunosuppressive treatment with tacrolimus. These data suggest that precursor cells are recruited early following transplantation to areas of donor vascular dysfunction in a process of attempted repair in the context of ongoing donor-recipient alloimmune interactions. Since cardiac TVD is primarily a vascular disorder affecting both intramyocardial and epicardial coronary arteries and veins ${ }^{14}$ and is characterized predominantly by EC damage and intimal proliferation, the results of this manuscript focus specifically on the role and contribution of recipient BM-derived cells in the repopulation of EC and SMC in the vasculature of cardiac TVD. However, there is also considerable interest and continued controversy surrounding the issue of whether BM-derived cells can directly or indirectly facilitate functional cardiac cell regeneration. Although the murine heterotopic heart transplant model is not the optimal model of cardiomyocyte injury to evaluate the potential rates of recipient BM-derived cells differentiation into cardiomyocytes, we have conducted preliminary studies and our results using desmin immunolabeling indicate that this phenomenon occurs at a very low frequency in this model. Additional studies will also be required to determine the degree to which other, non-BM-derived sources of recipient cell populations contribute to this replacement process and to identify the factors that modulate this process.

\section{Acknowledgements}

This work was funded by grants from the Canadian Institutes of Health Research (BMM, TJP) and the Heart and Stroke Foundation of British Columbia and Yukon (BMM). NR is the recipient of a Heart and Stroke Foundation of Canada Doctoral Research Award, the University of British Columbia University Graduate Fellowship and the Harry and Florence Dennison Fellowship in Medical Research.

\section{References}

1 Aranda Jr JM, Hill J. Cardiac transplant vasculopathy. Chest 2000;118:1792-1800.

2 Gao SZ, Schroeder JS, Alderman EL, et al. Clinical and laboratory correlates of accelerated coronary artery disease in the cardiac transplant patient. Circulation 1987;76:V56-V61.

3 Shimizu K, Sugiyama S, Aikawa M, et al. Host bone-marrow cells are a source of donor intimal smooth-muscle-like cells in murine aortic transplant arteriopathy. Nat Med 2001;7:738-741.

4 Sata M, Saiura A, Kunisato A, et al. Hematopoietic stem cells differentiate into vascular cells that participate in the pathogenesis of atherosclerosis. Nat Med 2002;8:403-409.

5 Saiura A, Sata M, Hirata Y, et al. Circulating smooth muscle progenitor cells contribute to atherosclerosis. Nat Med 2001;7:382-383.

6 Goodell MA, Brose K, Paradis G, et al. Isolation and functional properties of murine hematopoietic stem cells that are replicating in vivo. J Exp Med 1996;183: 1797-1806.

7 Corbel SY, Lee A, Yi L, et al. Contribution of hematopoietic stem cells to skeletal muscle. Nat Med 2003;9:1528-1532.

8 Jackson KA, Majka SM, Wang H, et al. Regeneration of ischemic cardiac muscle and vascular endothelium by adult stem cells. J Clin Invest 2001;107:1395-1402.

9 Quaini F, Urbanek K, Beltrami AP, et al. Chimerism of the transplanted heart. N Engl J Med 2002;346:5-15.

10 Laflamme MA, Myerson D, Saffitz JE, et al. Evidence for cardiomyocyte repopulation by extracardiac progenitors in transplanted human hearts. Circ Res 2002; 90:634-640.

11 Wright DE, Wagers AJ, Gulati AP, et al. Physiological migration of hematopoietic stem and progenitor cells. Science 2001;294:1933-1936.

12 Corry RJ, Winn HJ, Russell PS. Heart transplantation in congenic strains of mice. Transplant Proc 1973;5: 733-735.

13 Wellington CL, Walker EK, Suarez A, et al. ABCA1 mRNA and protein distribution patterns predict multiple different roles and levels of regulation. Lab Invest 2002;82:273-283.

14 Billingham ME. Pathology and etiology of chronic rejection of the heart. Clin Transplant 1994;8:289-292.

15 Hillebrands J, van den Hurk BM, Klatter FA, et al. Recipient origin of neointimal vascular smooth muscle cells in cardiac allografts with transplant arteriosclerosis. J Heart Lung Transplant 2000;19:1183-1192.

$16 \mathrm{Hu}$ Y, Davison F, Zhang Z, et al. Endothelial replacement and angiogenesis in arteriosclerotic lesions of 
allografts are contributed by circulating progenitor cells. Circulation 2003;108:3122-3127.

17 Hruban RH, Long PP, Perlman EJ, et al. Fluorescence in situ hybridization for the Y-chromosome can be used to detect cells of recipient origin in allografted hearts following cardiac transplantation. Am J Pathol 1993;142:975-980.

18 Atkinson C, Horsley J, Rhind-Tutt S, et al. Neointimal smooth muscle cells in human cardiac allograft coronary artery vasculopathy are of donor origin. J Heart Lung Transplant 2004;23:427-435.

19 Dong C, Wilson JE, Winters GL, et al. Human transplant coronary artery disease: pathological evidence for Fas-mediated apoptotic cytotoxicity in allograft arteriopathy. Lab Invest 1996;74:921-931.

20 Lai JC, Tranfield EM, Walker DC, et al. Ultrastructural evidence of early endothelial damage in coronary arteries of rat cardiac allografts. J Heart Lung Transplant 2003;22:993-1004.

$21 \mathrm{Hu}$ Y, Davison F, Ludewig B, et al. Smooth muscle cells in transplant atherosclerotic lesions are originated from recipients, but not bone marrow progenitor cells. Circulation 2002;106:1834-1839.

22 Hillebrands JL, Klatter FA, van den Hurk BM, et al. Origin of neointimal endothelium and alpha-actin- positive smooth muscle cells in transplant arteriosclerosis. J Clin Invest 2001;107:1411-1422.

$23 \mathrm{Hu} \mathrm{Y,} \mathrm{Mayr} \mathrm{M,} \mathrm{Metzler} \mathrm{B,} \mathrm{et} \mathrm{al.} \mathrm{Both} \mathrm{donor} \mathrm{and}$ recipient origins of smooth muscle cells in vein graft atherosclerotic lesions. Circ Res 2002;91:e13-20.

24 Jackson KA, Snyder DS, Goodell MA. Skeletal muscle fiber-specific green autofluorescence: potential for stem cell engraftment artifacts. Stem Cells 2004;22: 180-187.

25 Glaser R, Lu MM, Narula N, et al. Smooth muscle cells, but not myocytes, of host origin in transplanted human hearts. Circulation 2002;106:17-19.

26 Simper D, Wang S, Deb A, et al. Endothelial progenitor cells are decreased in blood of cardiac allograft patients with vasculopathy and endothelial cells of noncardiac origin are enriched in transplant atherosclerosis. Circulation 2003;108:143-149.

27 Li J, Han X, Jiang J, et al. Vascular smooth muscle cells of recipient origin mediate intimal expansion after aortic allotransplantation in mice. Am J Pathol 2001; 158:1943-1947.

28 Tanaka K, Sata M, Hirata Y, et al. Diverse contribution of bone marrow cells to neointimal hyperplasia after mechanical vascular injuries. Circ Res 2003;93: 783-790.

Supplementary Information accompanies the paper on Laboratory Investigation website (http://www.nature. com/labinvest) 